\title{
Revisiting the surface-energy-flux perspective on the sensitivity of global precipitation to climate change
}

\author{
Nicholas Siler · Gerard H. Roe · Kyle C. Armour · \\ Nicole Feldl
}

Received: date / Accepted: date

\begin{abstract}
Climate models simulate an increase in global precipitation at a rate of approximately $1-3 \%$ per Kelvin of global surface warming. This change is often interpreted through the lens of the atmospheric energy budget, in which the increase in global precipitation is mostly offset by an increase in net radiative cooling. Other studies have provided different interpretations from the perspective of the surface, where evaporation represents the turbulent transfer of latent heat to the atmosphere. Expanding on this surface perspective, here we derive a version of the Penman-Monteith equation that allows the change in ocean evaporation to be partitioned into a thermodynamic response to surface warming, and additional diagnostic contributions from changes in surface radiation, ocean heat uptake, and boundary-layer dynamics/relative humidity. In this framework, temperature is found to be the primary control on the rate of increase in global precipitation within model simulations of greenhouse gas warming, while the contributions from changes in surface radiation and ocean heat uptake are found to be secondary. The temperature contribution also dominates the spatial pattern of global evaporation change, leading to the largest fractional increases at high latitudes. In the surface energy budget, the thermodynamic increase in evaporation comes at the expense of the sensible heat flux, while radiative changes cause the sensible heat flux to increase. These tendencies on the sensible heat flux partly offset each other, resulting in a relatively small change in the global mean, and contributing to an impression that global precipitation is radiatively constrained.
\end{abstract}

Keywords Hydrologic Cycle · Global Warming

Nicholas Siler

College of Earth, Ocean, and Atmospheric Science, 104 CEOAS Admin Building, Oregon State University, Corvallis, OR 97331

E-mail: silern@oregonstate.edu

Gerard H. Roe

Department of Earth and Space Sciences, University of Washington, Seattle, Washington

Kyle C. Armour

School of Oceanography and Department of Atmospheric Sciences, University of Washington, Seattle, Washington

Nicole Feldl

Department of Earth and Planetary Sciences, University of California, Santa Cruz, Santa Cruz, California 


\section{Introduction}

In Earth's hydrologic cycle, water evaporates from the surface, condenses in the atmosphere, and returns to the surface as precipitation. It takes an average water molecule 8-10 days to complete this cycle (e.g., Van Der Ent and Tuinenburg, 2017). On timescales much longer than this, the rates of global-mean evaporation and precipitation are essentially equal. Because of the latent heat absorbed and released during evaporation and condensation, the hydrologic cycle plays an important role in the heat engine of the climate system, transferring energy from the warm surface, where most sunlight is absorbed, to the cooler atmosphere, where infrared radiation is emitted back to space.

In response to $\mathrm{CO}_{2}$-induced warming, climate models predict that the intensity of the global hydrologic cycle (i.e., global-mean evaporation/precipitation) will increase by around $1-3 \% / \mathrm{K}$, which is significantly less than the approximately $7 \% / \mathrm{K}$ increase in atmospheric water vapor resulting from the Clausius-Clapeyron equation and near-constant relative humidity (e.g., Boer, 1993; Allen and Ingram, 2002). This disparity has been invoked to explain important aspects of the climate response to $\mathrm{CO}_{2}$-induced warming, including a slowing down of the atmospheric circulation (Held and Soden, 2006), and an increase in the frequency and intensity of floods and droughts (Allen and Ingram, 2002; Trenberth, 1999, 2011). Understandably, therefore, the relationship between surface temperature and the global hydrologic cycle has been a central topic in climate science for decades (e.g., Manabe and Wetherald, 1975)

Many previous studies have investigated the change in global precipitation with warming from the perspective of the global-mean atmospheric energy budget,

$$
L \bar{P}=\overline{R_{a}}-\bar{H},
$$

where $L$ is the latent heat of vaporization, $P$ is the rate of precipitation, $R_{a}$ is the net heat lost through radiation, $H$ is the sensible heat flux from the surface to the atmosphere, and $(-)$ indicates the global mean of a quantity (e.g., Allen and Ingram, 2002; Stephens and Ellis, 2008; Previdi, 2010; Pendergrass and Hartmann, 2014; Fläschner et al, 2016). Eq. 1 implies that any increase in $L \bar{P}$ must be offset by a decrease in $\bar{H}$ and/or an increase in $\overline{R_{a}}$. In most simulations of $\mathrm{CO}_{2}$-induced warming by GCMs, the change in $\bar{H}$ tends to be small compared with changes in $L \bar{P}$ and $\overline{R_{a}}$, with the latter dominated by an increase in longwave emissions (e.g., Lambert and Webb, 2008; Pendergrass and Hartmann, 2014). This result has sometimes been interpreted as evidence that the change in global precipitation is primarily determined by the ability of the atmosphere to radiate more energy as it warms (e.g., Allen and Ingram, 2002). Yet in most cases, there is not a one-to-one trade-off between changes in $\overline{R_{a}}$ and $L \bar{P}$, because changes in $\bar{H}$ are often not negligible. For example, among GCMs participating in the most recent Coupled Model Intercomparison Project (CMIP5), Pendergrass and Hartmann (2014) found that the change in $\bar{H}$ was small but significant, with a magnitude about a third as large as the change in $L \bar{P}$. Simulations spanning a wider range of climate states have also been found to exhibit large variability in the change in $\bar{H}$ with global temperature (O'Gorman and Schneider, 2008; O'Gorman et al, 2012). On its own, however, the atmospheric energy budget provides limited insight into how the changes in $\bar{H}$, $L \bar{P}$, and $\overline{R_{a}}$ are partitioned.

An alternative to the atmospheric energy budget perspective is to treat the hydrologic cycle as a turbulence-driven process, in which evaporation, $E$, represents the turbulent flux of water vapor from the surface to the near-surface atmosphere (e.g., Penman, 1948; Priestly and Taylor, 1972; Monteith, 1981; Pierrehumbert, 2002; Richter and Xie, 2008; Lorenz et al, 
2010; Pierrehumbert, 2010). Over the oceans, where $85 \%$ of global evaporation occurs (e.g., Trenberth et al, 2007), the rate of evaporation is given approximately by the bulk transfer equation,

$$
E=\left[q^{*}\left(T_{s}\right)-r q^{*}\left(T_{a}\right)\right] \rho C_{H} u
$$

where $q^{*}$ is the specific humidity at saturation, $T_{s}$ and $T_{a}$ are the temperatures of the ocean surface and near-surface atmosphere, $r$ is the near-surface relative humidity, $\rho$ is the nearsurface air density, $C_{H}$ is the bulk transfer coefficient, and $u$ is the near-surface wind speed. Similarly, the sensible heat flux is approximately given by

$$
H=c_{p}\left(T_{s}-T_{a}\right) \rho C_{H} u,
$$

where $c_{p}$ is the specific heat capacity of air, and $C_{H}$ is typically assumed to have the same value as in Eq. 2. Eqs. 2 and 3 are derived from Monin-Obukhov similarity theory (e.g., Pierrehumbert, 2010), and they imply that the fluxes of latent and sensible heat are determined by the speed, temperature, and relative humidity of the near-surface winds, and by the difference in temperature between the ocean surface and the near-surface atmosphere. ${ }^{1}$ While these variables are all strongly dependent on atmospheric physics, collectively they must satisfy the surface energy budget,

$$
L E+H=R_{s}-G
$$

where $L, E$, and $H$ are defined as in Eq. $1, R_{S}$ is the net downward radiation flux at the surface, and $G$ is the rate of heat storage by the ocean plus-at high latitudes-the heat required to melt frozen hydrometeors that reach the surface. Unlike Eq. 1, Eqs. 2-4 are valid at a specific location, and not just in the global mean.

With three equations instead of one, the turbulent-flux perspective provides insight into the partitioning between $H$ and $L E$ that is not possible with the atmospheric energy budget alone. For example, Pierrehumbert (2002) has shown that one implication of Eqs. 2-4 is that $L E \ll H$ at very cold temperatures due to low values of $q^{*}$, while $L E \gg H$ at very warm temperatures due to high values of $q^{*}$, and correspondingly weak (or even negative) values of $T_{s}-T_{a}$, which are necessary to satisfy the surface energy budget (e.g., Le Hir et al, 2009). Interpolating between these two extremes, it must be true that, in more moderate climates like that of the present day, warming will tend to cause $L E$ to increase (via an increase in $q^{*}$ ), and $H$ to decrease (via a decrease in $T_{s}-T_{a}$ ). However, while such changes are easily diagnosed in GCM simulations (e.g., Richter and Xie, 2008; Lorenz et al, 2010), they are difficult to quantify from first principles, since they also depend on changes in net surface radiation, ocean heat uptake, and boundary-layer dynamics, which are only weakly constrained by Eqs. 2-4.

As we argue in this paper, however, the turbulent-flux perspective may have more explanatory power than previously assumed. By combining Eqs. 2-4 into a variant of the Penman-Monteith equation (Penman, 1948; Monteith, 1981), we show that the change in evaporation can be partitioned into four distinct terms, each with a straightforward physical interpretation. The first term depends only on surface temperature, and represents the thermodynamic response to warming, which is independent of other changes in the climate system. Meanwhile, the other terms represent the change in evaporation due to changes in surface radiation, ocean heat uptake, and boundary-layer dynamics/relative humidity. These terms cannot be derived from first principles, and must therefore be diagnosed from GCM

1 On long timescales, $T_{s}-T_{a}$ is generally positive over the ocean, implying a transfer of both sensible and latent heat from the surface to the atmosphere. 
simulations. Nevertheless, if the spatial patterns of changes in surface radiation and ocean heat uptake are known, the Penman-Monteith equation provides a way to quantify the resulting spatial pattern of the change in evaporation. When extended to the global mean, the Penman-Monteith framework also sheds light on the partitioning between changes in $\bar{H}$ and $L \bar{E}$ in the surface energy budget (or $L \bar{P}$ in the atmospheric energy budget) .

The paper is organized as follows. We begin in Section 2 by deriving a variant of the Penman-Monteith equation, which governs the rate of evaporation over the oceans subject to energetic constraints. In Section 3, we use this equation to diagnose the contributions to evaporation change in GCM simulations of global warming. In this diagnostic framework, we find that most of the increase in global evaporation is a direct consequence of warmer temperatures, while changes in surface radiation and ocean heat uptake play a secondary role. Based on these results, we then derive an approximation for evaporation change as a function of changes in temperature, surface radiation, and ocean heat uptake, and show that it accurately represents the fast and slow responses of evaporation to $\mathrm{CO}_{2}$ forcing, while also shedding light on the partitioning between changes in the latent and sensible heat flux. In Section 4, we apply the Penman-Monteith framework to a series of idealized simulations run by O'Gorman and Schneider (2008), and find that thermodynamics alone can account for much of the changes in global precipitation among the simulations. We conclude with a brief summary and discussion in Section 5.

\section{Derivation and interpretation of the Penman-Monteith equation.}

We build upon a long history of research on the physics of ocean evaporation, beginning with the fundamental equations (2-4) that govern the exchange of energy between the ocean surface and the atmosphere (Penman, 1948; Monteith, 1981; Pierrehumbert, 2002). In particular, we seek to solve this system of equations for $E$ while eliminating $T_{s}-T_{a}$ and $H$. To do so, we first approximate $q^{*}(T)$ as the first-order Taylor expansion about the point $T=T_{a}$ :

$$
q^{*}(T) \approx q^{*}\left(T_{a}\right)+\frac{d q^{*}}{d T}\left[T-T_{a}\right] .
$$

Given the Clausius-Clapeyron relation,

$$
\frac{d q^{*}}{d T}=\alpha q^{*}
$$

Eq. 5 implies that

$$
q^{*}\left(T_{s}\right) \approx q^{*}\left(T_{a}\right)\left(1+\alpha\left[T_{s}-T_{a}\right]\right)
$$

where

$$
\alpha=\frac{L}{R_{v} T_{a}^{2}}
$$

is the Clausius-Clapeyron scaling factor, with $R_{v}$ representing the specific gas constant for water vapor. Given Eq. 7, we can express the air-sea moisture difference in Eq. 2 as a function of $r, T_{a}$, and $T_{s}-T_{a}$ :

$$
E \approx q^{*}\left(T_{a}\right)\left(1-r+\alpha\left[T_{s}-T_{a}\right]\right) \rho C_{H} u .
$$

This allows us to eliminate $T_{s}-T_{a}$ and $H$ from Eqs. 2-4 to arrive at the following expression for the rate of evaporation from the ocean surface:

$$
L E \approx \eta\left(R_{s}-G+\kappa\right),
$$


where $\eta$ and $\kappa$ are defined as

$$
\eta \equiv \frac{1}{1+\beta_{0}},
$$

$$
\kappa \equiv(1-r) c_{p} \rho C_{H} u \alpha^{-1}
$$

with

$$
\beta_{0}=\frac{c_{p}}{\alpha L q^{*}\left(T_{a}\right)}
$$

representing the Bowen ratio $(H / L E)$ in the limit of $100 \%$ near-surface relative humidity $(r=1)$.

Eq. 10 is equivalent to the Penman-Monteith equation for terrestrial evapotranspiration from a saturated surface (i.e., where stomatal resistance is zero) (e.g., Scheff and Frierson, 2014). As written above, however, Eq. 10 is much easier to interpret than its more conventional form: besides $R_{S}$ and $G$, it comprises just two terms ( $\eta$ and $\kappa$ ), each of which has a straightforward physical meaning.

The first term, $\eta$, is a function of $T_{a}$ alone. In the context of climate change, therefore, $\eta$ captures the thermodynamic response of evaporation to warming, which is independent of changes in other variables like wind speed, relative humidity, and net surface radiation. On the other hand, $\eta$ does implicitly involve $T_{s}-T_{a}$ and $H$, as the following thought experiment illustrates.

Let us assume that $T_{s}$ and $T_{a}$ were to increase by the same amount, while all other variables in Eqs. 2-4 were held constant. In this scenario, $H$ would not change, while $L E$ would increase with $T_{a}$ at the same rate as $q^{*}\left(T_{a}\right)$-i.e., at the Clausius-Clapeyron rate of $\alpha \approx 7 \% / \mathrm{K}$ (Eq. 9; Richter and Xie, 2008). But without compensating changes in $R_{s}-G$, such an increase in $L E$ would clearly violate the surface energy budget. In reality, therefore, $T_{s}-T_{a}$ must decrease with warming, such that $H$ decreases and $L E$ increases at a rate less than $7 \% / \mathrm{K}$. This constraint on $T_{s}-T_{a}$ has been described in similar terms by Pierrehumbert (2002) and Lorenz et al (2010), but in Eq. 10, it is implicitly captured by a single variable, $\eta$.

Meanwhile, $\kappa$ represents the dependence of evaporation on relative humidity $(r)$ and boundary-layer dynamics ( $u$ and $C_{H}$ ), while its temperature-dependence (via $\alpha$ in its denominator) is negligibly small. An increase in $r$ will reduce the air-sea moisture difference, thereby causing $L E$ to decrease. Yet in the absence of changes in $R_{s}-G$, this decrease must be accompanied by an increase in $T_{s}-T_{a}$ to ensure no change in $L E+H$. Likewise, an increase in $u$ or $C_{H}$ will cause both $L E$ and $H$ to increase, and thus requires a simultaneous decrease in $T_{s}-T_{a}$ to satisfy the surface energy budget.

These thought experiments illustrate the crucial role played by $T_{s}-T_{a}$ within the PenmanMonteith framework (Eqs. 2-4). In response to any environmental change that, in isolation, would violate the surface energy budget, $T_{s}-T_{a}$ must adjust to conserve energy and, in the process, alter the partitioning between $L E$ and $H$. Physically, this framework is consistent with previous studies that have emphasized the role of $T_{s}-T_{a}$ in limiting the rate of increase in global precipitation with surface warming (Pierrehumbert, 2002; Richter and Xie, 2008; Lorenz et al, 2010). However, the advantage of the Penman-Monteith surface-energy perspective developed here is that it allows the change in evaporation with surface warming to be partitioned into a predictable component due to changes in $\eta$, and a diagnostic component due to changes in surface radiation, ocean heat uptake, and relative humidity/dynamics. In the next section, we show that this partitioning provides insight into the factors controlling the change in global and regional evaporation in response to $\mathrm{CO}_{2}$-induced warming. 


\section{Results from comprehensive GCMs}

In this section, we use the Penman-Monteith framework (Eq. 10) to quantify the factors contributing to the change in ocean evaporation in GCM simulations of $\mathrm{CO}_{2}$-induced warming. We examine both the equilibrium response to an abrupt doubling of atmospheric $\mathrm{CO}_{2}$, as simulated by an ensemble of atmosphere-only GCMs with slab oceans, and the transient response a century after an abrupt quadrupling of atmospheric $\mathrm{CO}_{2}$, as simulated by an ensemble of coupled atmosphere-ocean GCMs. The transient simulations were performed as part of the most recent Coupled Model Intercomparison Project (CMIP5; Taylor et al, 2012), while the equilibrium simulations were performed with a previous generation of climate models included in CMIP $3^{2}$ (Meehl et al, 2007). In the equilibrium (slab ocean) case, we focus on the change in ocean evaporation between the last five years of the pre-industrial control simulations and years 21-25 after $\mathrm{CO}_{2}$ doubling. The transient results reflect the change in evaporation between the pre-industrial control simulations and years 96-100 after $\mathrm{CO}_{2}$ quadrupling.

Tables 1 and 2 list the names of the 10 CMIP3 models and 12 CMIP5 models included in our analysis. Some models were excluded because the required variables were not readily available, or in the CMIP3 case, because the simulations were not in radiative equilibrium. For consistency with our later analysis in Section 3.1, we further restrict the CMIP5 models to those that also performed $\mathrm{CO}_{2}$ quadrupling simulations with prescribed climatological sea-surface temperatures (SSTs). The second column of each table gives the percent rate of change in global evaporation per Kelvin of global warming, while the third column gives the equivalent rate over the oceans (i.e., using ocean-mean instead of global-mean values of $\Delta E, E$, and $\Delta T)$. Even though the rate of change in ocean evaporation exceeds the global change in all models, the two values are highly correlated across models $(r=0.93$ and 0.81 for CMIP3 and CMIP5, respectively). This high correlation is not surprising, considering that the oceans account for $85 \%$ of global evaporation in the current climate (Trenberth et al, 2007), and an even larger share of the increase in evaporation under global warming (e.g., Fu and Feng, 2014). To first order, therefore, we can understand the change in global evaporation (and thus precipitation) by focusing on the ocean-mean changes.

Fig. 1 shows the fractional change in ocean evaporation per Kelvin of local warming in the ensemble mean of the equilibrium (left column) and transient (right column) simulations, along with the individual contributions to evaporation change due to changes in $\eta, R_{S}, G$, and $\kappa$ (rows 2-5). We have calculated these contributions using the discrete form of the fractional derivative of Eq. 10 with respect to $T_{a}$ :

$$
\frac{1}{\Delta T_{a}}[\overbrace{\frac{\Delta E}{E}}^{1} \approx \overbrace{\frac{\Delta \eta}{\eta}}^{2}+\frac{\eta}{L E}(\overbrace{\Delta R_{s}}^{3}-\overbrace{\Delta G}^{4}+\overbrace{\Delta \kappa}^{5})],
$$

where $\Delta$ indicates the change in a variable between the control and warmed climate. The number above each term in Eq. 14 indicates its order, from top to bottom, in Fig. 1. Each contribution was calculated using monthly-mean model output (see Appendix for details). The ocean-mean of each contribution is given in the top left corner of each panel. Columns 4-7 of Tables 1 and 2 give the equivalent values for each ensemble member, along with the standard deviation of each contribution across the ensemble. In this Section we discuss only the ensemble-mean results (Fig. 1), but will address the inter-model variability in Section 5.

\footnotetext{
${ }^{2}$ Equilibrium simulations were not part of CMIP5.
} 
In both the equilibrium and transient cases, we find that $\Delta \eta$ accounts for the largest contribution to evaporation change, increasing from about $1.2 \% / \mathrm{K}$ at the equator to more than $4 \% / \mathrm{K}$ at higher latitudes. To understand this meridional structure-which is also reflected in the pattern of total evaporation change-consider the analytic expression for the $\Delta \eta$ contribution, which can be derived from Eqs. 6, 8, 11, and 13:

$$
\frac{d \ln \eta}{d T_{a}}=\frac{\beta_{0}}{1+\beta_{0}}\left(\alpha-\frac{2}{T_{a}}\right)
$$

Because $\beta_{0}$ decreases almost exponentially with increasing temperature, $d \ln \eta / d T_{a}$ is largest at cold temperatures where it approaches $\left(\alpha-2 / T_{a}\right)$-not much less than the ClausiusClapeyron scaling of atmospheric water vapor under fixed relative humidity. As $T_{a}$ increases, however, the gap between $\alpha$ and $d \ln \eta / d T_{a}$ grows ever larger. The increase in $d \ln \eta / d T_{a}$ with latitude therefore reflects the meridional structure of $T_{a}$ in the control climate. In the global mean, $\Delta \eta$ causes an increase in evaporation of about $1.5 \%$ per Kelvin of global warming in both the equilibrium and transient cases, accounting for the largest fraction of the total increase. ${ }^{3}$

Compared with $\Delta \eta$, the contributions from the other terms in Eq. 14 are generally smaller in magnitude. $\Delta R_{s}$ accounts for the second largest contribution to evaporation change (Fig. 1, row 3 ), increasing evaporation by about $1 \% / \mathrm{K}$ globally in both the equilibrium and transient cases due to an increase in net surface radiation in the tropics and midlatitudes. ${ }^{4}$ In contrast, the contribution from $\Delta G$ is negligible in the equilibrium (slab-ocean) simulations, but significantly negative in the transient simulations due to ocean heat uptake in the subpolar North Atlantic Ocean and Southern Ocean (row 4). Finally, the contribution from $\Delta \kappa$ tends to be negative but quite weak (row 5), implying that changes in boundary-layer dynamics and relative humidity play a minor role, particularly in determining the spatial pattern of evaporation change.

Fig. 1 reveals two key points about how ocean evaporation responds to $\mathrm{CO}_{2}$-induced warming in GCMs. First, the largest contribution to evaporation change comes from $\Delta \eta$, which is a direct consequence of warmer temperatures (Eq. 15). This means that much of the increase in ocean evaporation with warming - both globally and regionally-is independent of the nature of both the forcing that drove the temperature change (e.g., aerosols vs. greenhouse gases) and how certain physical processes are parameterized within a model (e.g., boundary-layer dynamics, convection, clouds, etc.).

Second, the small contribution from $\Delta \kappa$ suggests that changes in boundary layer dynamics and relative humidity play a limited role in determining the change in ocean evaporation in response to $\mathrm{CO}_{2}$-induced warming, particularly at regional scales. We know of no a priori reason why this should be the case, since in principle the boundary layer could adjust in any number of ways that would alter the surface energy balance (e.g., Pierrehumbert, 2002). However, if we take as given that the contribution from $\Delta \kappa$ is small, the total change in evaporation can then be approximated as

$$
L \Delta E \approx L E \frac{d \ln \eta}{d T_{a}} \Delta T_{a}+\eta \Delta\left(R_{s}-G\right)
$$

\footnotetext{
3 The global-mean contributions represent the average of the fractional changes (Fig. 1), weighted by the product of mean-state evaporation and local temperature change. Further details are provided in the Appendix.

4 The contribution from $\Delta R_{S}$ is negative at high latitudes in the Southern Hemisphere, reflecting a decrease in shortwave absorption as a result of increased cloud cover.
} 
where the first term represents the thermodynamic response to warming (Eq. 15), and the second term represents the diagnostic component of evaporation change due to the combined changes in surface radiation and ocean heat uptake.

\subsection{Testing Eq. 16 on the fast and slow responses of evaporation to $\mathrm{CO}_{2}$ forcing.}

Although $\Delta \kappa$ has little impact on evaporation in Fig. 1, it is reasonable to question the generality of this result. For example, it could be that $\Delta \kappa$ is not physically independent of the other terms in Eq. 14, in which case its small contribution in Fig. 1 might reflect a cancellation between larger competing effects driven by changes in $\eta$ and $R_{s}-G$. If true, this would imply that Eq. 16 does not generally hold.

To test the robustness of the approximation in Eq. 16, therefore, we revisit the coupled CMIP5 simulations analyzed previously, but now with the change in evaporation partitioned into "fast" and "slow" components that represent, respectively, the direct response of evaporation to $\mathrm{CO}_{2}$ quadrupling with fixed SSTs, and the more gradual changes that occur as the climate warms. Following previous studies (e.g., Lambert and Faull, 2007; Lambert and Webb, 2008; Lambert et al, 2009; Andrews et al, 2009, 2010; Andrews and Forster, 2010; Frieler et al, 2011; Samset et al, 2016; Fläschner et al, 2016), we define the fast response as the change that occurs when $\mathrm{CO}_{2}$ is quadrupled and SSTs are fixed at pre-industrial values (i.e., the difference between sstClim4xCO2 and sstClim experiments in CMIP5 parlance), and the slow response as the difference between the coupled 4xCO2 simulations and the fixed-SST 4xCO2 simulations. Defined in this way, the sum of the slow and fast responses gives the total change in the top right panel of Fig. 1.

In the fast response to $\mathrm{CO}_{2}$ forcing, an increase in the longwave optical depth of the atmosphere will cause an increase in net surface radiation (e.g., Allen and Ingram, 2002; McInerney and Moyer, 2012) and a decrease in outgoing longwave radiation. The latter effect adds heat to the climate system, which is mostly absorbed by the ocean (thus increasing $G$ ). Meanwhile, little warming occurs over the oceans because $T_{S}$ is held constant, implying that the first term on the RHS of Eq. 16 will be small. As a result, the fast change in evaporation is approximately given by

$$
L \Delta E_{\text {fast }} \approx \eta \Delta\left(R_{s}-G\right)_{\text {fast }},
$$

where $\Delta\left(R_{S}-G\right)_{\text {fast }}$ represents the change in $R_{S}-G$ that is directly caused by $\mathrm{CO}_{2}$ forcing, independent of surface warming.

On longer timescales, $T_{a}$ will gradually rise, impacting ocean evaporation in two ways. First, there will be a thermodynamic response represented by the first term on the RHS of Eq. 16. Second, $R_{S}$ and $G$ will also change as the atmosphere warms and the earth approaches top-of-atmosphere radiative balance (e.g., Pierrehumbert, 1999). Applying the regression method of Gregory et al (2004) at each grid point, we find that the slow change in $R_{s}-G$ over most of the global oceans is well approximated in all models as a linear surface-temperature feedback,

$$
\Delta\left(R_{s}-G\right)_{\text {slow }} \approx \lambda \Delta T_{a},
$$

where $\lambda$ represents the slope of the regression. The slow response of evaporation can then be expressed as

$$
L \Delta E_{\text {slow }} \approx\left(L E \frac{d \ln \eta}{d T_{a}}+\eta \lambda\right) \Delta T_{a}
$$


Fig. 2 shows the approximations in Eqs. 17 and 19 (top row) along with the actual fast and slow changes in evaporation from the ensemble mean of the CMIP5 simulations (bottom row). Comparing the two rows, we find that the approximations capture the spatial pattern of evaporation change remarkably well, with spatial correlations of $r>0.99$ in both cases. The approximations are also quite accurate at the global scale, deviating from the actual change in ocean-mean evaporation by $16 \%$ and $4 \%$ in the fast and slow cases, respectively. ${ }^{5}$ These results show that the contribution from $\Delta \kappa$ is small even when $\Delta\left(R_{S}-G\right)$ is independent of surface warming, suggesting that Eq. 16 is robust within GCM simulations of $\mathrm{CO}_{2}$-induced warming.

3.2 Implications of the Penman-Monteith perspective for changes in the sensible heat flux.

Having demonstrated the approximate validity of Eq. 16, let us now consider its implications for the change in sensible heat flux $(\Delta H)$. When combined with the surface energy budget (Eq. 4), Eq. 16 implies that

$$
\Delta H \approx-L E \frac{d \ln \eta}{d T_{a}} \Delta T_{a}+(1-\eta) \Delta\left(R_{s}-G\right)
$$

which can be further decomposed into fast and slow components, following the same line of reasoning used to derive Eqs. 17 and 19:

$$
\Delta H_{\text {fast }} \approx(1-\eta) \Delta\left(R_{S}-G\right)_{\text {fast }},
$$

$$
\Delta H_{\text {slow }} \approx\left(-L E \frac{d \ln \eta}{d T_{a}}+(1-\eta) \lambda\right) \Delta T_{a} .
$$

These equations provide insight into how $L \Delta E$ and $\Delta H$ are partitioned in the fast and slow responses to $\mathrm{CO}_{2}$ warming. In the fast case, Eqs. 17 and 21 imply that

$$
\frac{\Delta H_{\text {fast }}}{L \Delta E_{\text {fast }}} \approx \frac{1-\eta}{\eta}=\beta_{0}
$$

which is a function of $T_{a}$ alone (Eq. 13). In the current climate, $\beta_{0}$ ranges from around 0.25 in the tropical warm pool to more than 1.5 at high latitudes, with an ocean-mean value of around 0.5 . Even though this result is specific to the oceans, it helps explain why in the fast response to $\mathrm{CO}_{2}$ forcing, $L \Delta \bar{E}$ significantly exceeds $\Delta \bar{H}$ in the global mean (e.g., Bala et al, 2008).

In the slow case, $L \Delta E$ (Eq. 19) and $\Delta H$ (Eq. 22) both consist of two terms: one stemming from a change in $\eta$, and the other from the slow change in $R_{s}-G$ (i.e., $\lambda \Delta T_{a}$ ). Fig. 3 shows the contributions from these terms in the ensemble mean of the CMIP5 simulations. The $\Delta \eta$ terms (top row) are equal and opposite to each other, representing an increase in $L E$ at the expense of $H$. In contrast, both $\lambda \Delta T_{a}$ terms (second row) are generally positive, with magnitudes that differ by a factor of $\beta_{0}$, mirroring the response to $\Delta\left(R_{s}-G\right)_{\text {fast }}$ (Eq. 23 ). When these contributions are combined (third row), the positive tendencies on $L \Delta E$ reinforce each other, while the opposing tendencies on $\Delta H$ mostly cancel. This explains why ocean-mean $\Delta H$ is close to zero in the slow response to $\mathrm{CO}_{2}$ forcing (bottom row;

5 These percentages are based on a comparison of the ocean-mean values that appear in the top left of each panel in Fig. 2. 
Andrews et al, 2009), and why it is significantly smaller in magnitude than ocean-mean $L \Delta E$ in the total (fast + slow) response, where $\Delta\left(R_{S}-G\right)$ is also generally positive.

This result points to an important conceptual difference between the Penman-Monteith perspective and other interpretations of evaporation change based on diagnostic assessments of the surface or atmospheric energy budgets. In the surface energy budget, the relatively small decrease in $H$ over the global oceans implies that the increase in $L E$ is mostly offset by an increase in $R_{S}-G$. Similarly, in the global-mean atmospheric energy budget, the small magnitude of $\Delta \bar{H}$ means that $L \Delta \bar{P}$ is mostly offset by $\Delta \overline{R_{a}}$ (Eq. 1).

Within the Penman-Monteith framework, however, Fig. 1 shows that $\Delta\left(R_{s}-G\right)$ accounts for just $45 \%$ and $41 \%$ of the change in ocean-mean evaporation in the equilibrium and transient simulations, respectively - a very different diagnosis than suggested by the surface energy budget alone. This difference stems from the fact that, in addition to the surface energy budget, the Penman-Monteith equation also incorporates other physical constraints related to the turbulent transfer of latent and sensible heat from the surface (Eqs. 2-3). In particular, $\eta$ represents a thermodynamic constraint on the partitioning between $L E$ and $H$, as noted in Section 2. When this constraint is combined with energy conservation, $\Delta H$ and $L \Delta E$ are found to be closely related, each responding to $\Delta T_{a}$ and $\Delta\left(R_{s}-G\right)$ according to Eqs. 16 and 20. In GCM simulations, $\Delta T_{a}$ and $\Delta\left(R_{s}-G\right)$ conspire to make $\Delta H$ relatively small over most of the oceans, but this is by no means a general result. In a much cooler climate, for example, Eq. 20 indicates that $\Delta H$ would be significantly larger in response to a similar amount of warming.

\section{Thermodynamic constraints on global precipitation over a wide range of climates.}

In the previous Section, we showed that the change in ocean evaporation in simulations of $\mathrm{CO}_{2}$-induced warming can be separated into a thermodynamic component that is a direct consequence of surface warming, and a diagnostic component that represents the effects of changes in net surface radiation, ocean heat uptake, and boundary-layer dynamics/relative humidity. Of these, the thermodynamic component was found to account for $2 / 3$ of the total increase in ocean evaporation in equilibrium slab-ocean simulations, and for an even larger share in transient coupled simulations. The thermodynamic component is also strongly dependent on mean-state temperature, explaining why the largest fractional increase in evaporation tends to occur at high latitudes in Fig. 1. In light of these results, it is worth considering how thermodynamics might influence global precipitation over a wide range of climates much warmer and cooler than our own.

For this portion of our analysis, we revisit a series of idealized gray-radiation simulations run by O'Gorman and Schneider (2008), which were designed to exhibit a wide range of global-mean surface temperatures in response to imposed changes in atmospheric longwave optical depth, albeit with no representation of the radiative effects of clouds and atmospheric water vapor. Since the output from these simulations is not publicly available, we are unable to diagnose the factors contributing to their differences in global-mean precipitation, as we did for the CMIP ensembles. However, we can estimate the thermodynamic contribution to these differences by assuming that $\eta$ varies with global-mean surface temperature (Eq. $11)$, and that all other variables are approximately constant $\left(R_{s}-G+\kappa=197 \mathrm{Wm}^{-2}\right.$; see Appendix). This gives the following approximation for global precipitation as a function of global-mean surface temperature alone (Eq. 10):

$$
L \bar{P} \approx \eta\left(\overline{T_{a}}\right) \times 197 \mathrm{Wm}^{-2} .
$$


Fig. 4 shows the estimated global precipitation based on Eq. 24 (gray line) compared with the actual results of O'Gorman and Schneider (2008) (black x's). Despite some discrepancies at low temperatures, the agreement is good overall, suggesting that most of the change in global precipitation among their idealized simulations can be explained as a direct consequence of the change in global-mean surface temperature.

Of course, the real world is more complicated than the idealized, gray-radiation GCM used by O'Gorman and Schneider (2008). This might explain why changes in surface radiation appear to be smaller in these simulations than in the more realistic CMIP simulations discussed previously.

Yet even if surface radiation is not constant, it is still instructive to consider how global precipitation varies with surface temperature as a consequence of thermodynamics alone. Taking the derivative of $\eta$ with respect to $T_{a}$, we find that the slope of the $\bar{P}$-vs. $-\overline{T_{a}}$ curve in Fig. 4 is proportional to

$$
\frac{d \bar{P}}{d T_{a}} \propto \frac{\beta_{0}}{\left(1+\beta_{0}\right)^{2}}\left(\alpha-\frac{2}{\overline{T_{a}}}\right) .
$$

Importantly, this equation encapsulates what Pierrehumbert (2002) has identified as distinct constraints on global precipitation operating in different temperature regimes. At the cold extreme, $\beta_{0} \gg 1$ as $q^{*}$ approaches 0 . While this results in a large fractional increase in global precipitation with warming (Eq. 15), the actual increase is small (Eq. 25), reflecting the atmosphere's limited capacity at cool temperatures to maintain water vapor against condensation. Conversely, at very warm temperatures, $\beta_{0} \ll 1$ due to high values of $q^{*}$. In this regime, atmospheric water vapor is plentiful, but the change in global precipitation with warming is limited by net radiation at the surface (Eq. 4). It is therefore between these limits, where $\beta_{0}$ is $\mathrm{O}(1)$ (i.e., $T_{a} \approx 280 \mathrm{~K}$ ), that global precipitation is thermodynamically most sensitive to changes in global mean surface temperature. While changes in surface radiation will affect the upper bound on global precipitation in the warm limit, the broad shape of the $\bar{P}$-vs. $-\overline{T_{a}}$ curve is guaranteed by thermodynamic constraints inherent in $\eta$.

Finally, it is interesting to consider how the primacy of $\eta$ in GCM simulations of $\mathrm{CO}_{2}$ induced warming relates to two other ideas for how global precipitation changes with warming. The first, put forth by Kleidon and Renner $(2013 \mathrm{a}, \mathrm{b})$, is that the hydrologic-cycle heat engine operates near the thermodynamic limit of maximum power. Using an idealized energy balance model, Kleidon and Renner (2013b) explore the implications of this assumption for how global evaporation/precipitation scales with warming, assuming no change in net surface radiation. Consistent with Eq. 24, they find that global evaporation is proportional to a term that can be shown to be identical to $\eta$, except that $\overline{T_{a}}$ in their model represents the average temperature at which water vapor condenses in the atmosphere. This suggests that Kleidon and Renner's foundational assumption-that atmospheric convection approaches the thermodynamic limit of maximum power-may indeed have some relevance to GCM simulations, and perhaps also to the real atmosphere.

A second, similarly idealized conceptualization of the hydrologic cycle was proposed by Takahashi (2009), who argued that the change in precipitation with global warming is controlled by radiative cooling from the free troposphere rather than the full atmosphere. This idea-which represents a variant of the atmospheric energy budget perspective-is based on the principle that little of the surface sensible heat flux makes it out of the marine boundary layer. Using a 1-dimensional radiative-convective-equilibrium model constrained by this and a few other a priori assumptions, Takahashi found changes in precipitation with warming that were similarly consistent with the idealized results of O'Gorman and Schneider in Fig. 4 (O'Gorman et al, 2012). While the idealized models of Takahashi (2009) and Kleidon 
and Renner (2013a,b) do not appear to be incompatible, it remains unclear how they might relate to each other, or to the Penman-Monteith framework presented in this paper. We hope that future research will shed light on this important question.

\section{Discussion}

In this paper, we have shown that the Penman-Monteith equation, as expressed in Eq. 10, allows any change in ocean evaporation to be partitioned into distinct contributions from changes in surface temperature, net surface radiation, ocean heat uptake, and boundary layer dynamics/relative humidity. In GCM simulations of $\mathrm{CO}_{2}$-induced warming, we find that the majority of the change in ocean evaporation is a direct consequence of warming, represented by $\Delta \eta$ in Eq. 14. This component of evaporation change derives from fundamental thermodynamics, and therefore does not depend on the specific nature of the radiative forcing or on the model physics. Physically, this term represents a change in the partitioning between latent and sensible heat fluxes due to an increase in the surface-air moisture gradient (required by the Clausius-Clapeyron equation), and a corresponding decrease in the surface-air temperature gradient (required by energy conservation). In fractional terms, the change in evaporation due to this effect diminishes with warming, explaining why the largest fractional changes in ocean evaporation tend to occur at high latitudes in GCM simulations (Fig. 1). Compared with this thermodynamic effect, the contribution to evaporation change from changes in net surface radiation $\left(\Delta R_{S}\right)$ and ocean heat uptake $(\Delta G)$ were found to be secondary but still significant, while changes in boundary-layer dynamics and relative humidity $(\Delta \kappa)$ were found to be less important, particularly at regional scales.

Because $\Delta \kappa$ is small, the Penman-Monteith framework allows the change in evaporation to be estimated from the spatial pattern of $\Delta T_{a}, \Delta R_{s}$, and $\Delta G$ alone (Eq. 16). For example, in the fast response to $\mathrm{CO}_{2}$ forcing, $R_{S}-G$ decreases due to significant ocean heat uptake, causing a decrease in global evaporation (and thus precipitation). Because SSTs are fixed, this decrease in evaporation is well approximated as $\eta \Delta\left(R_{S}-G\right)$. On longer timescales, surface temperatures rise and evaporation increases, in part due to thermodynamics, and in part because ocean heat uptake declines as the climate system returns to radiative equilibrium. Combined with the surface energy budget, Eq. 16 also leads to an equally accurate approximation of the change in sensible heat flux, $H$ (Eq. 20). Thus, from the Penman-Monteith perspective, $L E$ and $H$ represent two sides of the same coin, each responding to $\Delta T_{a}, \Delta R_{s}$, and $\Delta G$ according to Eqs. 16 and 20.

This interpretation of global hydrologic change is somewhat different from those based on the atmospheric energy budget, in which the change in global precipitation $(L \Delta \bar{P})$ is offset by $\Delta \bar{H}$ and a change in net atmospheric radiative cooling $\left(\Delta \overline{R_{a}}\right)$. The energy-budget perspective provides little insight into $\Delta \bar{H}$, but has much to say about the physics behind $\Delta \overline{R_{a}}$ (e.g., Lambert and Webb, 2008; Stephens and Ellis, 2008; Previdi, 2010; Pendergrass and Hartmann, 2014; DeAngelis et al, 2015; Fläschner et al, 2016). In contrast, the PenmanMonteith equation provides new insight into the partitioning between $L \Delta E$ and $\Delta H$ over the oceans (Eqs. 16 and 20), but only if $\Delta T_{a}$ and $\Delta\left(R_{s}-G\right)$ (which is equal to $\Delta R_{a}$ in the global mean) are already known. This shows that the energy-budget and Penman-Monteith perspectives are fully complementary, and together provide a more complete understanding of evaporation change than either can provide by itself.

The Penman-Monteith perspective may also shed light on the response of the global hydrologic cycle to a change in the solar constant (e.g., Wetherald et al, 1975; Andrews et al, 2009), to changes in radiation due to solar geoengineering (Bala et al, 2008), or to 
non-greenhouse forcings like a volcanic eruption (Trenberth and Dai, 2007). Of course, the accuracy of Eq. 16 is contingent on the forcing having little impact on the dynamics or relative humidity of the atmospheric boundary layer. In certain scenarios-e.g., a change in the concentration of absorbing aerosols in the boundary layer (Ming et al, 2010; Samset et al, 2016) - this condition might not be met. Yet even in these cases, the Penman-Monteith framework could prove to be a powerful tool for diagnosing the various contributions to changes in ocean evaporation at both global and regional scales.

Finally, it is important to note that while changes in surface radiation are of secondary importance to the overall change in ocean evaporation, they account for most of the intermodel spread, as evidenced by the standard deviations in the bottom row of Tables 1 and 2. Relative to the other terms, the standard deviation of the $\Delta R_{S}$ contribution is roughly 2-3 times larger across both the equilibrium and transient ensembles. This is not surprising given that $\Delta \overline{R_{s}}$ is closely tied to $\Delta \overline{R_{a}}$ (Eqs. 1 and 4), which depends on several model variables that are not well constrained, including clouds, tropospheric humidity, and the radiative transfer parameterization for calculating shortwave absorption by water vapor (DeAngelis et al, 2015; Fläschner et al, 2016). In contrast, the $\Delta \eta$ contribution is more consistent due to broad model agreement in the spatial patterns of warming, mean-state temperature, and mean-state evaporation. Altogether, these results suggest that thermodynamics alone will contribute to an increase in global precipitation with surface warming at a rate of about $1.5 \% / \mathrm{K}$; whether global precipitation increases at a rate closer to 1 or $3 \% / \mathrm{K}$ will largely depend on radiative changes.

Acknowledgements We are very grateful to Ray Pierrehumbert and four anonymous reviewers for their excellent comments that greatly improved the paper.

\section{A Calculating the contributions to evaporation change in Eq. 14.}

The terms in Eq. 14 were calculated as follows: $L E$ and $R_{s}$ were taken directly from model output; $G$ was determined from $R_{s}, L E$, and $H$ based on the surface energy budget (Eq. 4); $\eta$ was calculated from the twometer air temperature using Eqs. 11 and 13. Finally, given $L E, \eta, R_{s}$, and $G$, we then solved for $\kappa$ in Eq. 10. The contributions were calculated from ensemble-mean output over the last five years of the simulation period. In the equilibrium warming simulations, this was typically $21-25$ years after $\mathrm{CO}_{2}$ doubling. In the transient warming simulations, we used years $96-100$ after $\mathrm{CO}_{2}$ quadrupling. The contributions were first calculated for each month, and then the monthly contributions were averaged to arrive at an annual-mean value. However, the results were essentially unchanged when the contributions were calculated from annualmean output.

To understand the global impact of the fractional contributions in Fig. 1, we must account for spatial variability in the magnitude of the mean-state evaporation and surface-air warming. To do so, we multiply each term in Eq. 14 by the following (dimensionless) weighting function,

$$
w=\frac{E \Delta T_{a}}{\bar{E} \Delta \overline{T_{a}}},
$$

where the overbars in the denominator indicate the ocean-mean values of each variable. These results are then averaged in space, yielding the ocean-mean contributions given in the top left of each panel in Fig. 1.

\section{B Estimating $R_{S}-G+\kappa$ in the idealized simulations of O'Gorman and Schneider (2008).}

To estimate the value of $R_{S}-G+\kappa$ in O'Gorman and Schneider's (2008) simulations, we use the fact that their control climate exhibits a global-mean surface-air temperature of $\overline{T_{a}}=288 \mathrm{~K}$, and a global-mean precipitation 
of $4.3 \mathrm{~mm} /$ day, which equates to $L \bar{E}=124 \mathrm{Wm}^{-2}$. Given $\eta \approx 0.63$ at $T=288 \mathrm{~K}$, this implies a combined value of $R_{s}-G+\kappa=197 \mathrm{Wm}^{-2}$. If we assume that this sum is constant, global precipitation is directly proportional to $\eta$, resulting in the gray curve in Fig. 4.

\section{References}

Allen M, Ingram WJ (2002) Constraints on future changes in climate and the hydrologic cycle. Nature 419(6903):224-232

Andrews T, Forster PM (2010) The transient response of global-mean precipitation to increasing carbon dioxide levels. Environmental Res Lett 5(2):025,212, DOI 10.1088/17489326/5/2/025212

Andrews T, Forster PM, Gregory JM (2009) A Surface Energy Perspective on Climate Change. J Climate 22(10):2557-2570, DOI 10.1175/2008JCLI2759.1

Andrews T, Forster P, Boucher O, Bellouin N, Jones A (2010) Precipitation, radiative forcing and global temperature change. Geophys Res Lett 37(14):L14,701, DOI 10.1029/2010GL043991

Bala G, Duffy PB, Taylor KE (2008) Impact of geoengineering schemes on the global hydrological cycle. Proc Natl Acad Sci U S A 105(22):7664-9, DOI 10.1073/pnas.0711648105

Boer G (1993) Climate change and the regulation of the surface moisture and energy budgets. Climate Dynamics 8:225-239

DeAngelis AM, Qu X, Zelinka MD, Hall A (2015) An observational radiative constraint on hydrologic cycle intensification. Nature 528(7581):249-253, DOI 10.1038/nature15770

Fläschner D, Mauritsen T, Stevens B (2016) Understanding the Intermodel Spread in GlobalMean Hydrological Sensitivity*. J Climate 29(2):801-817, DOI 10.1175/JCLI-D-150351.1

Frieler K, Meinshausen M, Schneider von Deimling T, Andrews T, Forster P (2011) Changes in global-mean precipitation in response to warming, greenhouse gas forcing and black carbon. Geophys Res Lett 38(4):n/a-n/a, DOI 10.1029/2010GL045953

Fu Q, Feng S (2014) Responses of terrestrial aridity to global warming. J Geophys Res: Atmos 119(13):7863-7875, DOI 10.1002/2014JD021608

Gregory JM, Ingram WJ, Palmer MA, Jones GS, Stott PA, Thorpe RB, Lowe JA, Johns TC, Williams KD (2004) A new method for diagnosing radiative forcing and climate sensitivity. Geophysical Research Letters 31(3):L03,205, DOI 10.1029/2003GL018747

Held IM, Soden BJ (2006) Robust Responses of the Hydrological Cycle to Global Warming. J Climate 19(21):5686-5699

Kleidon A, Renner M (2013a) A simple explanation for the sensitivity of the hydrologic cycle to surface temperature and solar radiation and its implications for global climate change. Earth System Dynamics 4(2):455-465, DOI 10.5194/esd-4-455-2013

Kleidon A, Renner M (2013b) Thermodynamic limits of hydrologic cycling within the Earth system: concepts, estimates and implications. Hydrol Earth Syst Sci 17(7):2873-2892, DOI 10.5194/hess-17-2873-2013

Lambert FH, Faull NE (2007) Tropospheric adjustment: The response of two general circulation models to a change in insolation. Geophys Res Lett 34(3):L03,701, DOI 10.1029/2006GL028124

Lambert FH, Webb MJ (2008) Dependency of global mean precipitation on surface temperature. Geophys Res Lett 35(16):L16,706, DOI 10.1029/2008GL034838 
Lambert FH, Allen MR, Lambert FH, Allen MR (2009) Are Changes in Global Precipitation Constrained by the Tropospheric Energy Budget? J Climate 22(3):499-517, DOI 10.1175/2008JCLI2135.1

Le Hir G, Donnadieu Y, Goddéris Y, Pierrehumbert RT, Halverson GP, Macouin M, Nédélec A, Ramstein G (2009) The snowball Earth aftermath: Exploring the limits of continental weathering processes. Earth and Planetary Science Letters 277(3-4):453-463, DOI 10.1016/j.eps1.2008.11.010

Lorenz DJ, DeWeaver ET, Vimont DJ (2010) Evaporation Change and Global Warming: The Role of Net Radiation and Relative Humidity. J Geophys Res: Atmos 115(D20):D20,118, DOI 10.1029/2010JD013949

Manabe S, Wetherald RT (1975) The Effects of Doubling the CO 2 Concentration on the climate of a General Circulation Model. J Atmos Sci 32(1):3-15, DOI 10.1175/15200469(1975)032;0003:TEODTC $\dot{i} 2.0 . \mathrm{CO} ; 2$

McInerney D, Moyer E (2012) Direct and disequilibrium effects on precipitation in transient climates. Atmospheric Chemistry and Physics Discussions 12(8):19,649-19,681, DOI 10.5194/acpd-12-19649-2012, URL http://www.atmos-chemphys-discuss.net/12/19649/2012/

Meehl GA, Covey C, Delworth T, Latif M, McAvaney B, Mitchell JFB, Stouffer RJ, Taylor KE, Meehl GA, Covey C, Delworth T, Latif M, McAvaney B, Mitchell JFB, Stouffer RJ, Taylor KE (2007) THE WCRP CMIP3 Multimodel Dataset: A New Era in Climate Change Research. Bull Amer Meteor Soc 88(9):1383-1394, DOI 10.1175/BAMS-88-91383

Ming Y, Ramaswamy V, Persad G (2010) Two opposing effects of absorbing aerosols on global-mean precipitation. Geophys Res Lett 37(13):L13,701, DOI 10.1029/2010GL042895

Monteith JL (1981) Evaporation and surface temperature. Q J Royal Met Soc 107(451):127, DOI 10.1002/qj.49710745102

O'Gorman PA, Schneider T (2008) The Hydrological Cycle over a Wide Range of Climates Simulated with an Idealized GCM. J Climate 21(15):3815-3832, DOI 10.1175/2007JCLI2065.1

O'Gorman PA, Allan RP, Byrne MP, Previdi M (2012) Energetic Constraints on Precipitation Under Climate Change. Surveys in Geophysics 33(3-4):585-608, DOI 10.1007/s10712-011-9159-6

Pendergrass AG, Hartmann DL (2014) The Atmospheric Energy Constraint on Global-Mean Precipitation Change. J Climate 27(2):757-768, DOI 10.1175/JCLI-D-13-00163.1

Penman HL (1948) Natural Evaporation from Open Water, Bare Soil and Grass. Proceedings of the Royal Society A: Mathematical, Physical and Engineering Sciences 193(1032):120-145, DOI 10.1098/rspa.1948.0037

Pierrehumbert RT (1999) Subtropical water vapor as a mediator of rapid global climate change. Mechanisms of Global Climate Change at Millennial Time Scales pp 339-361, DOI 10.1029/GM112p0339

Pierrehumbert RT (2002) The hydrologic cycle in deep-time climate problems. Nature 419(6903):191-8, DOI 10.1038/nature01088

Pierrehumbert RT (2010) Principles of planetary climate. Cambridge University Press

Previdi M (2010) Radiative feedbacks on global precipitation. Environment Res Lett 5(2):025,211, DOI 10.1088/1748-9326/5/2/025211

Priestly CHB, Taylor RJ (1972) On the Assessment of Surface Heat Flux and Evaporation Using Large-Scale Parameters. Mon Wea Rev 100(2):81-92, DOI 10.1175/15200493(1972)100;0081:OTAOSH $; 2.3 . C O ; 2$ 
Richter I, Xie SP (2008) Muted precipitation increase in global warming simulations: A surface evaporation perspective. J Geophys Res: Atmos 113(D24):D24,118, DOI 10.1029/2008JD010561

Samset BH, Myhre G, Forster PM, Hodnebrog $\varnothing$, Andrews T, Faluvegi G, Fläschner D, Kasoar M, Kharin V, Kirkevåg A, Lamarque JF, Olivié D, Richardson T, Shindell D, Shine KP, Takemura T, Voulgarakis A (2016) Fast and slow precipitation responses to individual climate forcers: A PDRMIP multimodel study. Geophys Res Lett 43(6):27822791, DOI 10.1002/2016GL068064

Scheff J, Frierson DMW (2014) Scaling Potential Evapotranspiration with Greenhouse Warming. J Climate 27(4):1539-1558, DOI 10.1175/JCLI-D-13-00233.1

Stephens GL, Ellis TD (2008) Controls of Global-Mean Precipitation Increases in Global Warming GCM Experiments. J Climate 21(23):6141-6155, DOI 10.1175/2008JCLI2144.1

Takahashi K (2009) Radiative Constraints on the Hydrological Cycle in an Idealized RadiativeConvective Equilibrium Model. J Atmos Sci 66(1):77-91, DOI 10.1175/2008JAS2797.1

Taylor KE, Stouffer RJ, Meehl GA, Taylor KE, Stouffer RJ, Meehl GA (2012) An Overview of CMIP5 and the Experiment Design. Bull Amer Meteor Soc 93(4):485-498, DOI 10.1175/BAMS-D-11-00094.1

Trenberth K (1999) Conceptual framework for changes of extremes of the hydrological cycle with climate change. Climatic Change 42(1):327-339, DOI 10.1023/a:1005488920935

Trenberth K (2011) Changes in precipitation with climate change. Climate Research 47(1):123-138, DOI 10.3354/cr00953

Trenberth KE, Dai A (2007) Effects of Mount Pinatubo volcanic eruption on the hydrological cycle as an analog of geoengineering. Geophysical Research Letters 34(15), DOI 10.1029/2007GL030524

Trenberth KE, Smith L, Qian T, Dai A, Fasullo J, Trenberth KE, Smith L, Qian T, Dai A, Fasullo J (2007) Estimates of the Global Water Budget and Its Annual Cycle Using Observational and Model Data. J Hydrometeor 8(4):758-769, DOI 10.1175/JHM600.1

Van Der Ent RJ, Tuinenburg OA (2017) The residence time of water in the atmosphere revisited. Hydrol Earth Syst Sci 21:779-790, DOI 10.5194/hess-21-779-2017

Wetherald RT, Manabe S, Wetherald RT, Manabe S (1975) The Effects of Changing the Solar Constant on the Climate of a General Circulation Model. J Atmos Sci 32(11):2044-2059, DOI 10.1175/1520-0469(1975)032;2044:TEOCTS $i 2.0 . C O ; 2$ 
Table 1 First column: The names of the CMIP3 (slab-ocean) models included in our analysis. Second column: The rate of increase in global-mean evaporation in response to a doubling of atmospheric $\mathrm{CO}_{2}$, after reaching radiative equilibrium. Third column: The rate of increase in ocean-mean evaporation. Columns 4-7: The individual contributions to changes in ocean-mean evaporation from $\Delta \eta, \Delta R_{s}, \Delta G$, and $\Delta \kappa$, according to Eq. 14. The second row from the bottom gives the ensemble-mean rates (Fig. 1). These were calculated from ensemble-mean variables, and therefore differ slightly from the average rates of the individual models. The bottom row gives the standard deviation across models. In the second (third) column, global-mean (oceanmean) rates were calculated using the global-mean (ocean-mean) values of $\Delta E, E$, and $\Delta T$. Global-mean and ocean-mean rates are highly correlated at $r=0.93$, indicating the dominant influence of the ocean on global evaporation.

\begin{tabular}{l|c|c|c|c|r|r}
\hline \hline CMIP3 model & $\frac{\Delta E}{E \Delta T}$ (globe) & $\frac{\Delta E}{E \Delta T}$ (oceans) & $\Delta \eta$ & $\Delta R_{s}$ & $\Delta G$ & $\Delta \kappa$ \\
\hline Can-CGCM3.1 (T47) & 2.02 & 2.14 & 1.37 & 0.96 & -0.01 & -0.22 \\
Can-CGCM3.1 (T63) & 2.18 & 2.28 & 1.37 & 0.95 & 0.07 & -0.16 \\
CSIRO-Mk3.0 & 2.26 & 2.67 & 1.56 & 1.20 & -0.01 & -0.13 \\
GFDL-CM2 & 1.35 & 1.76 & 1.53 & 0.59 & 0.06 & -0.43 \\
HadGEM1 & 1.81 & 2.18 & 1.48 & 0.89 & 0.09 & -0.34 \\
INM-CM3 & 1.58 & 1.85 & 1.56 & 0.71 & -0.06 & -0.37 \\
MIROC3.2 (hires) & 1.92 & 2.13 & 1.47 & 1.05 & 0.02 & -0.46 \\
MIROC3.2 (medres) & 2.18 & 2.37 & 1.51 & 1.23 & -0.01 & -0.39 \\
MPI-OM & 1.95 & 2.23 & 1.46 & 1.07 & -0.10 & -0.23 \\
MRI-CGCM2 & 2.28 & 2.48 & 1.52 & 1.16 & 0.01 & -0.24 \\
\hline Ensemble mean & 1.97 & 2.22 & 1.49 & 1.00 & 0.01 & -0.30 \\
Standard deviation & 0.30 & 0.27 & 0.07 & 0.21 & 0.06 & 0.12 \\
\hline
\end{tabular}

Table 2 As in Table 1, but for the CMIP5 (coupled) simulations. The rates of change in evaporation are based on years 96-100 after $\mathrm{CO}_{2}$ quadrupling. The global-mean and ocean-mean rates are correlated at $r=0.81$.

\begin{tabular}{l|c|c|c|c|c|c}
\hline \hline CMIP5 model & $\frac{\Delta E}{E \Delta T}$ (globe) & $\frac{\Delta E}{E \Delta T}$ (oceans) & $\Delta \eta$ & $\Delta R_{s}$ & $\Delta G$ & $\Delta \kappa$ \\
\hline BCC-CSM1.1 & 1.65 & 1.98 & 1.41 & 1.00 & -0.21 & -0.26 \\
CanESM2 & 1.41 & 1.85 & 1.44 & 0.74 & -0.19 & -0.17 \\
CCSM4 & 1.46 & 1.99 & 1.42 & 1.07 & -0.22 & -0.34 \\
CSIRO-Mk3.6.0 & 1.85 & 2.29 & 1.53 & 1.29 & -0.27 & -0.35 \\
HadGEM2-ES & 1.23 & 1.78 & 1.43 & 0.92 & -0.22 & -0.42 \\
INM-CM4 & 1.35 & 1.58 & 1.54 & 0.79 & -0.46 & -0.31 \\
IPSL-CM5A-LR & 2.02 & 2.64 & 1.71 & 1.53 & -0.27 & -0.38 \\
MIROC5 & 1.46 & 1.64 & 1.46 & 1.02 & -0.34 & -0.59 \\
MPI-ESM-LR & 1.63 & 2.19 & 1.52 & 1.20 & -0.34 & -0.24 \\
MPI-ESM-MR & 1.77 & 2.26 & 1.49 & 1.21 & -0.27 & -0.23 \\
MRI-CGCM3 & 2.27 & 2.27 & 1.43 & 1.28 & -0.29 & -0.23 \\
NorESM1-M & 1.43 & 1.96 & 1.52 & 1.12 & -0.32 & -0.40 \\
\hline Ensemble mean & 1.62 & 2.04 & 1.51 & 1.10 & -0.28 & -0.33 \\
Standard deviation & 0.30 & 0.31 & 0.08 & 0.22 & 0.08 & 0.11 \\
\hline
\end{tabular}



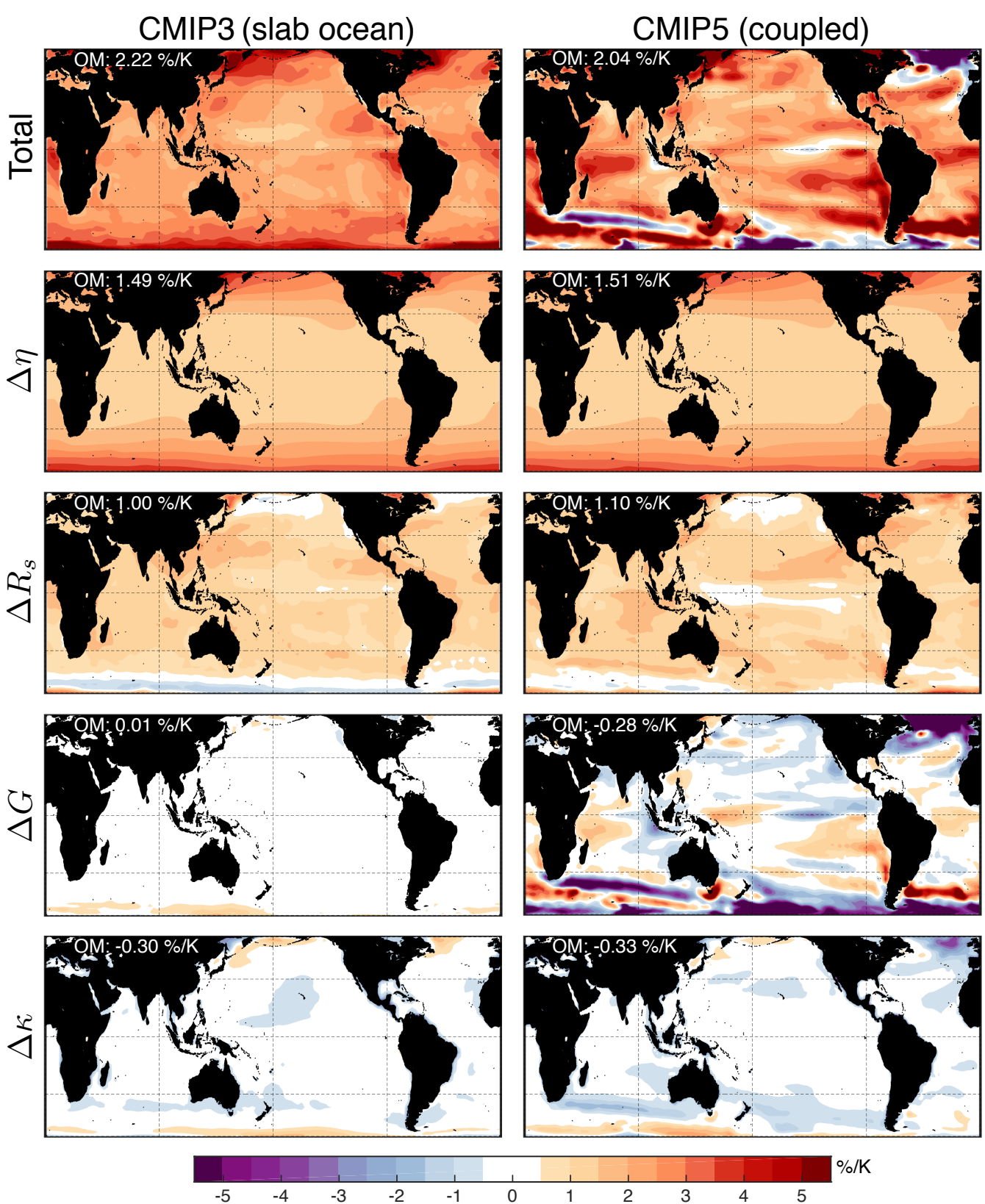

Fig. 1 The percent change in evaporation over the oceans in response to $\mathrm{CO}_{2}$-induced warming (top row), and the individual contributions from changes in $\eta$ (second row), $R_{s}$ (third row), $G$ (fourth row), and $\kappa$ (fifth row), in the equilibrium (left column) and transient (right column) simulations. Each contribution was calculated from ensemble-mean output according to Eq. 14 (see Appendix), and represents the change per Kelvin of global warming over the oceans, which is equal to $3.15 \mathrm{~K}$ in the $2 \times \mathrm{CO}_{2}$ equilibrium simulations, and $4.13 \mathrm{~K}$ in the $4 \times \mathrm{CO}_{2}$ transient simulations. The top left corner of each panel gives the ocean-mean (OM) values of each contribution. The results are broadly similar between the equilibrium and transient ensembles, with the exception of the contribution from ocean-heat uptake $(\Delta G)$, which is negligible in the equilibrium simulations due to the absence of a dynamical ocean. 

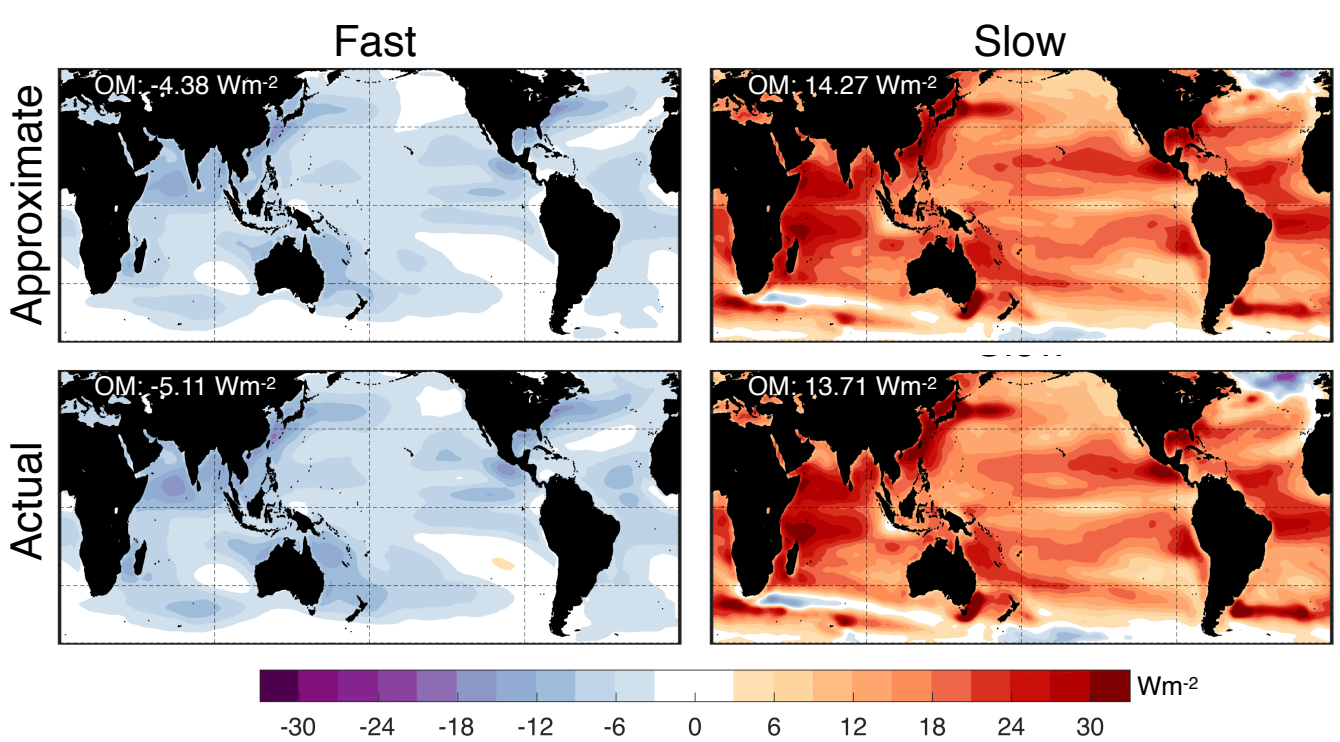

Fig. 2 The "fast" (left column) and "slow" (right column) changes in ocean evaporation in GCM simulations of an abrupt quadrupling of atmospheric $\mathrm{CO}_{2}$ (in $\mathrm{Wm}^{-2}$ ). The fast component represents the direct response of evaporation to $\mathrm{CO}_{2}$ quadrupling with fixed SSTs, while the slow component represents the gradual changes that occur as the climate warms. Top row: the approximate changes calculated from Eqs. 17 and 19, using ensemble-mean values of $F, S, E, T_{a}$, and $\Delta T_{a}$. Bottom row: the actual changes in the ensemble mean of CMIP5 simulations. Ocean-mean (OM) values are given in the top left corner of each panel. 

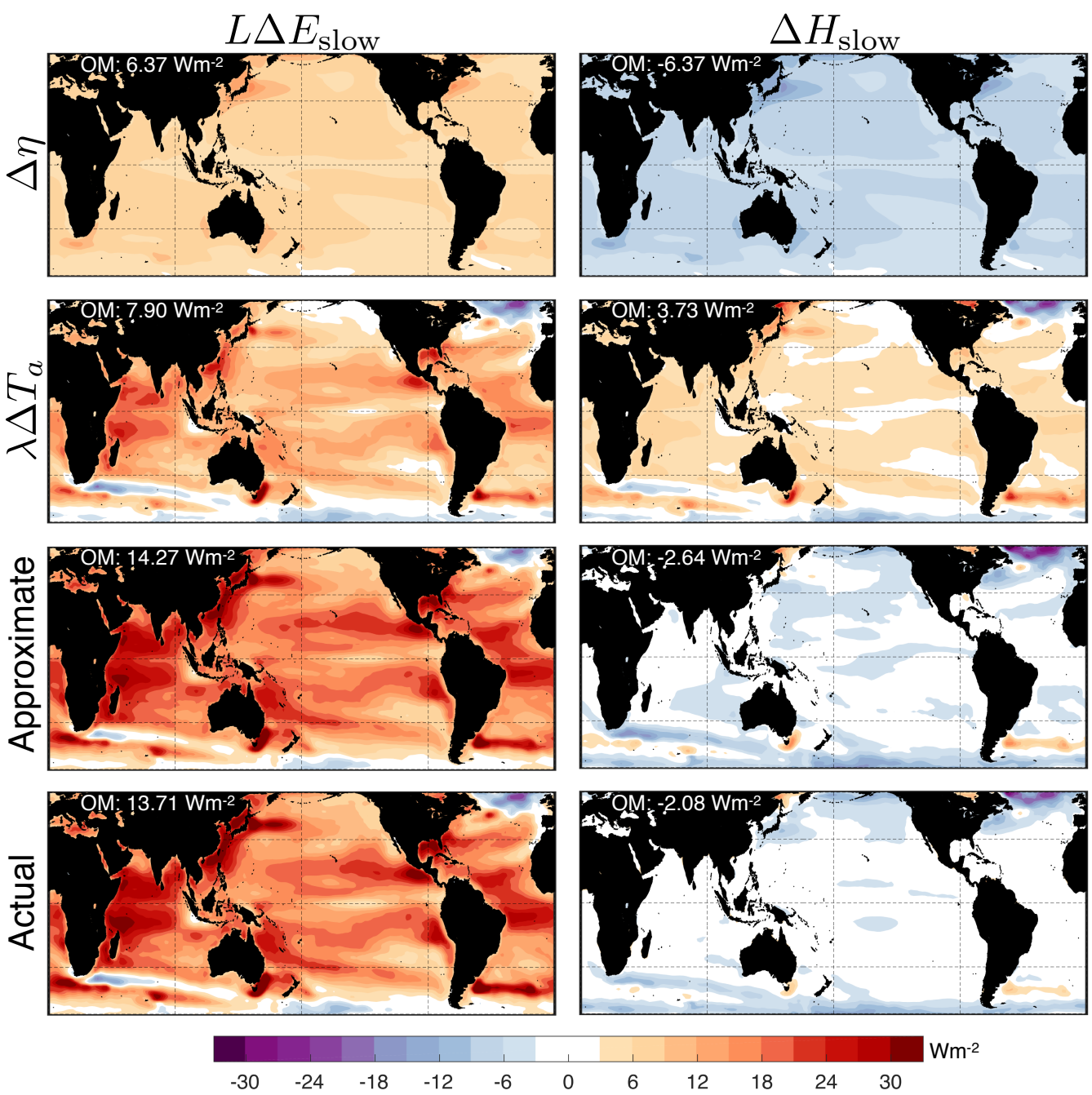

Fig. 3 The slow changes in $L E$ (left column) and $H$ (right column) in GCM simulations of an abrupt quadrupling of atmospheric $\mathrm{CO}_{2}$ (in $\mathrm{Wm}^{-2}$ ). Top row: the contribution from the $\Delta \eta$ term in Eqs. 16 and 20 . Second row: the contribution from the $\lambda \Delta T_{a}$ term in Eqs. 16 and 20. Third row: the full approximation given by Eqs. 16 and 20. Fourth row: the actual change in the ensemble mean of CMIP5 simulations. Note that the two panels on the bottom left match those in the right column of Fig. 2. 


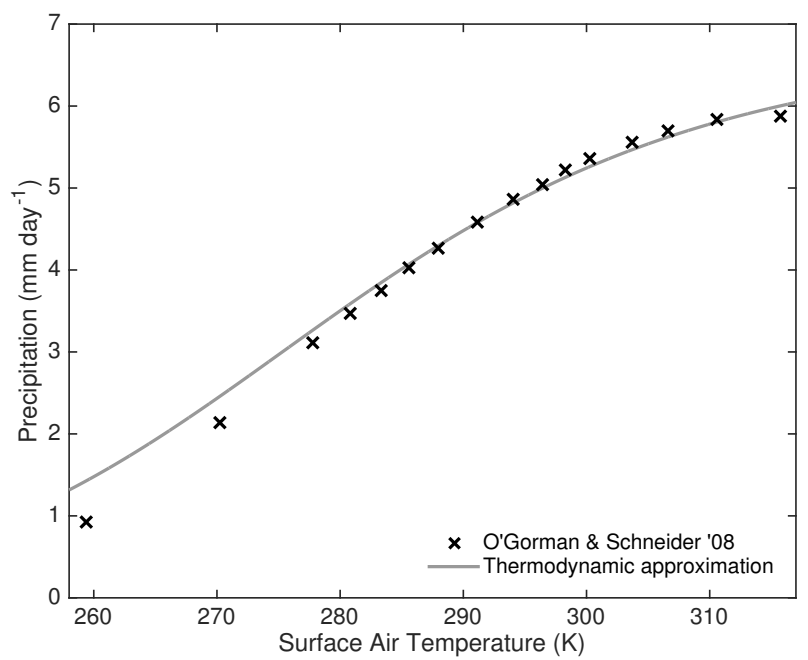

Fig. 4 Global mean precipitation $(P)$ as a function of global-mean surface-air temperature $\left(\Delta \overline{T_{a}}\right)$, according to the idealized simulations of O'Gorman and Schneider (2008) (black X's), and the thermodynamic approximation assuming that $\eta$ varies with global-mean surface temperature and $R_{S}-G+\kappa$ is held fixed at 197 $\mathrm{Wm}^{-2} \mathrm{~K}^{-1}$ (gray line). 